\title{
The Processing and Representation of Dutch and English Compounds: Peripheral Morphological and Central Orthographic Effects
}

\author{
Nivja H. de Jong,* Laurie B. Feldman, $†$ Robert Schreuder,* Matthew Pastizzo, $\dagger$ \\ and R. Harald Baayen* \\ *Interfaculty Research Unit for Language \& Speech, and Max Planck Institute for Psycholinguistics, \\ Nymegen, The Netherlands; and †The University at Albany, State University of New York, and \\ Haskins Laboratories
}

Published online December 6, 2001

\begin{abstract}
In this study, we use the association between various measures of the morphological family and decision latencies to reveal the way in which the components of Dutch and English compounds are processed. The results show that for constituents of concatenated compounds in both languages, a position-related token count of the morphological family plays a role, whereas English open compounds show an effect of a type count, similar to the effect of family size for simplex words. When Dutch compounds are written with an artificial space, they reveal no effect of type count, which shows that the differential effect for the English open compounds is not superficial. The final experiment provides converging evidence for the lexical consequences of the space in English compounds. Decision latencies for English simplex words are better predicted from counts of the morphological family that include concatenated and hyphenated but not open family members. (c) 2001 Elsevier Science (USA)
\end{abstract}

Several frequency effects have been reported in the domain of word recognition. The string frequency of the presented word itself (the surface frequency) as well as the summed frequency of all its inflectional variants (the base frequency) have been found to influence response latencies (e.g., Taft 1979; Baayen, Dijkstra, \& Schreuder 1997).

Another effect which has recently been found to play a role in lexical processing is a type count effect: the morphological family size. The morphological family of a monomorphemic word consists of all words containing that word as a morpheme. For Dutch simplex words and for stems in derived words, participants respond faster in visual lexical decision to words with large families than to words with small families (matched for surface and base frequency). The summed frequencies of the morphological family members, the family frequency, does not influence reaction times (Schreuder \& Baayen, 1997; De Jong, Schreuder, \& Baayen, 2000). For English, Baayen, Lieber, and Schreuder (1997) showed that simplex nouns with a high family frequency are rated equally high in a subjective frequency rating as nouns with a low family frequency, but nouns with a high family size are rated higher than nouns

This study was financially supported by the Dutch National Research Council NWO (PIONIER grant to the fifth author), the University of Nijmegen (The Netherlands), and the Max Planck Institute for Psycholinguistics (Nijmegen, The Netherlands).

Address correspondence and reprint requests to Nivja de Jong, Max Planck Institute for Psycholinguistics, P.O. Box 310, 6500 AH Nijmegen, The Netherlands. E-mail: nivja.dejong@mpi.nl. 
with a low family size. We have now replicated these results using the visual lexical decision task: The same stimuli as used by Baayen et al. (1997) again showed an effect of family size and an absence of an effect of family frequency. ${ }^{1}$

There are three independent kinds of evidence suggesting that the family size effect permeates semantic levels of lexical processing. First, the family size effect occurs in tasks requiring central levels of processing (visual lexical decision and subjective frequency rating) but not in a task tapping into form-related stages of lexical processing such as visual progressive demasking (Schreuder \& Baayen, 1997). Second, the effect is not mediated by form. Dutch irregular past participles which differ in their orthographic and phonological form from their morphological family members nevertheless show an effect of family size. Furthermore, in the case that the stem-allomorph used in the irregular past participle is by itself a word with a different meaning (e.g., the noun vocht, "moisture,' is embedded in the past participle gevochten, "fought," which is derived from vechten, "to fight"), a count of morphological family members of such a form-related but not semantically related embedded word does not influence response latencies (De Jong et al., 2000). Third, removing opaque family members from the count of family size enhances correlations with reaction times (Bertram, Baayen, \& Schreuder, 2000).

In the present study, we use variables characterizing the morphological family of the constituents to investigate how compounds are processed and stored. In the literature on the processing of compounds in Dutch, two contrasting results have been reported. On the one hand, Van Jaarsveld and Rattink (1988) report frequency effects for compounds, and the absence of a frequency effect for the constituents of these compounds. These results suggest that compounds are accessed as wholes. For a similar view with respect to compounds in English and other languages, see MarslenWilson (2001), but see Taft and Forster (1976). On the other hand, several semantic priming studies have shown that both constituents of semantically transparent (and to some extent opaque) compounds can be primed (Sandra, 1990; Zwitserlood, 1994). ${ }^{2}$ Similar contrasting results have been reported for comparatives in Dutch, for which the frequency of the base form appears to be irrelevant (Bertram, Schreuder, \& Baayen, 2000) while at the same time, the family size of the base predicts response latencies (Bertram, Baayen, \& Schreuder, 2000). Given these results for Dutch comparatives, the present article investigates whether the family size of the constituent affects the response latencies. If so, this would provide further evidence that the constituents of compounds play a role in the processing of compounds.

Indeed, the first experiment reported below revealed the anticipated outcome: larger left or right constituent family sizes led to shorter reaction times. However, post hoc analyses show that a different interpretation of the results is called for. The position family frequency, the family frequency of a constituent constrained by position within the compound, is a better predictor of reaction times than is the family size of the constituent. We argue that this position family frequency effect is a diagnostic of peripheral on-line decomposition of the compound and that, nevertheless, the frequency of the constituent itself is irrelevant.

\footnotetext{
${ }^{1}$ We conducted two experiments (visual lexical decision: see the procedure reported for Experiment 4 of the present article; 17 participants each), using the materials from Experiments 2 and 3 of Baayen et al. (1997). The words with a high family frequency were responded to equally fast as the words with a low family frequency ( 661 and $649 \mathrm{~ms}$ respectively: $t 1, t 2<1$ ), but the words with large morphological families were responded to faster than words with small families [643 and 699 ms respectively: $t 1(16)=-3.17, p=.003 ; t 2(40)=-2.71, p=.005]$.

${ }^{2}$ To eliminate possible confounds with semantic transparency, we used only semantically reasonably transparent compounds in the present study. Further research is required to investigate possible differences in processing the constituents of opaque and transparent compounds with respect to variables referring to the morphological family.
} 
Experiment 2 replicates the position family frequency effect for English compounds, but only for those compounds which are written without an intervening space (henceforth concatenated compounds). For compounds written with a space between the constituents (henceforth open compounds), this experiment revealed a position family size effect rather than a position family frequency effect. Experiment 3 shows that the insertion of an artificial space into Dutch compounds does not lead to a position family size effect. Experiment 4, finally, shows that English open compounds do not belong to the morphological families of simplex words, providing converging evidence that the different kinds of orthographic conventions of English compounds correlate with different kinds of central representations.

\section{EXPERIMENT 1}

In two subexperiments, we investigate the role of the family size of constituents in Dutch compounds using standard visual lexical decision. In post hoc analyses, we investigate the influence of several frequency counts. In Experiment 1a, we contrast the family size of the left constituent. In Experiment 1b, we contrast the right constituent family size.

\section{Method}

Participants. Twenty-four participants responded to the set of compounds of Experiment 1a, and another 24 participants responded to the set of compounds of Experiment 1b. Most participants were undergraduates at Nijmegen University and all were native speakers of Dutch.

Materials of Experiment 1a. We selected 112 transparent Dutch compounds from the CELEX lexical database (Baayen, Piepenbrock, \& Gulikers, 1995). None of these compounds contained a linking morpheme, ${ }^{3}$ and all were compounds composed of two nouns. We built a contrast in family size of the left constituent, while keeping the right constituent constant (e.g., the pair ijzerwinkel, "hardware store," and antiekwinkel, "antique shop"). Fifty-six left constituents had a mean family size of 52 (30), and the mean family size of the 56 left constituents with a small family was 7 (4) (standard deviations between parentheses). The compounds were matched on the frequency of the left constituent ${ }^{4}$ [high: mean 29.0 (27.8); low: mean 27.2 (28.2)], the compound frequency [high: mean 0.5 (0.5); low: mean 0.5 (0.6)], and for mean length in letters (high: 10.1; low: 10.5). We did not match for the left constituent family frequency, as previous studies had shown that the family frequency does not affect response latencies.

Materials of Experiment $1 \mathrm{~b}$. We selected a different set of 112 transparent Dutch compounds from the CELEX lexical database. None of these compounds contained a linking morpheme and all were noun-noun compounds. Fifty-six of these compounds had a right constituent with a high family size such as molen in windmolen, "windmill" [mean: 55 (41)], and 56 compounds with identical left constituents had a right constituent with a low family size such as vlaag in windvlaag, "gust of wind" [mean: 7 (4)]. We matched the compounds on the frequency of the right constituent [high: mean 33.2 (25.8); low: mean: 31.9 (30.2)], compound frequency [high: mean 0.5 (0.8); low: mean $0.5(0.6)$ ], and mean length in letters (high: 10.6; low:11.3). Note that the properties of these compounds are comparable to the properties of the compounds used in Experiment 1a, including the contrast in family size for the left (Experiment 1a) and right (Experiment 1b) constituent. As in Experiment 1a, we did not match for the (right) constituent family frequency, as previous studies had shown that the family frequency does not affect response latencies.

For each experiment, we constructed two lists such that the same constituent occurred in one list only and such that the number of compounds falling into the two family size conditions were evenly distributed. Each compound was paired with a pseudocompound, which consisted of an existing constituent

\footnotetext{
${ }^{3}$ In Dutch, compounds can occur with the linking morpheme -en- or $-s$ - between the constituents or without a linking morpheme.

${ }^{4}$ Throughout this article, the frequency of a noun (either monomorphemic or compound) is the summed frequency of all its inflectional variants. But note that, in addition to matching for this frequency, we always also matched on the singular form. All frequency counts are standardized per million.
} 
at either the left or right position combined with a pseudoconstituent which did not violate the phonotactical rules of Dutch.

Procedure. Participants were tested in noiseproof experimental rooms. They were asked to decide as quickly and accurately as possible whether a letter string appearing on the computer screen was a real Dutch word. Each stimulus was preceded by a fixation mark in the middle of the screen for 500 ms. After $50 \mathrm{~ms}$, the stimulus appeared at the same position. Stimuli were presented on Nec Multisync color monitors in white lowercase 21-point Helvetica letters on a dark background and they remained on the screen for $1500 \mathrm{~ms}$. The maximum time span allowed for a response was $2000 \mathrm{~ms}$ from stimulus onset.

\section{Results and Discussion}

The participants performed both experiments with an overall error rate that was less than 20\%. In Experiment 1a, the mean reaction time of one item in the high condition (propagandaliteratuur, "propaganda literature") differed by more than 3 standard deviations from the mean reaction time and was excluded from further analyses. Its matched pair (bekentenisliteratuur, "confessional literature") was also excluded from further analyses. Remaining items were entered into analyses by subjects $(t 1)$ and by items $(t 2)$. The mean reaction time for the high condition in Experiment 1a was significantly shorter than the mean reaction time of the low condition [660 (101) and 698 (96) ms respectively: $t 1(23)=-6.09, p=.000 ; t 2(108)=-2.47$, $p=.000]$ and elicited fewer erroneous responses [0.04 (0.04) and $0.09(0.08)$ error proportions respectively; $t 1(23)=-3.85, p=.001 ; t 2(108)=-2.30, p=.012]{ }^{5}$ For Experiment $1 \mathrm{~b}$, we also find an effect of $38 \mathrm{~ms}$ between the high and low condition [674 (111) and 712 (129) ms respectively: $t 1(23)=-5.49, p=.000$; $t 2(110)=-2.23, p=.014]$ and a difference in error proportions, although not reliably so in the by-item analysis $[0.06(0.06)$ and $0.09(0.06)$ error proportions respectively: $t 1(23)=-2.51, p=.010 ; t 2(110)=-1.09, p=.140]$.

A post hoc analysis of Experiments $1 \mathrm{a}$ and $1 \mathrm{~b}$ revealed that in addition to the family size of both the left and right constituents the compound frequency was an important factor in determining response latencies. ${ }^{6}$ The correlation between compound frequency and reaction times for the words in Experiment 1a was $r=$ $-.383[t(108)=-4.31, p=.000]$ and for the words in Experiment $1 \mathrm{~b} r=-.347$ $[t(110)=-3.89, p=.000]$. Further correlation analyses revealed two important results. First, the correlation of the position family size with reaction times in both experiments was higher than or comparable to the correlation of the family size. The position family of a constituent in a compound consists of a count of family members in which the constituent appears at the same position as it does in the target compound. For a constituent such as molen, "mill,"' in windmolen, the position family would include family members such as watermolen, "water mill,', and koffiemolen, " coffee grinder," but not a word such as molensteen, "'millstone." Second, the correlation of the summed frequencies of the position family members (the position family frequency) was higher than the correlation of the position family size with reaction times.

In previous studies (Schreuder \& Baayen, 1997; De Jong et al., 2000), the family frequency never played a significant role. In post hoc correlation studies, a significant correlation might be obtained for the family frequency, but this correlation would always be lower than the correlation with family size and could always be accounted for as being a spurious correlation resulting from the high intercorrelation between

\footnotetext{
${ }^{5}$ Throughout this article we report one-sided $t$ tests (except when mentioned otherwise), as the studied effects are expected to be facilitatory.

${ }^{6}$ Throughout this article, all correlation analyses are carried out with log-transformations on all frequencies and family size counts.
} 
family size and family frequency. In the present experiments, however, the correlation for the position family frequency is higher than the correlation for the position family size, suggesting that this position family frequency cannot be a mere spurious correlation. Instead of reporting in detail the subtle differences in these correlations, we constructed, after having pooled all the compounds of both experiments, four post hoc factorial designs. In the first design, we contrasted the left position family frequency; in the second we contrasted right position family frequency; in the third, left position family size; and in the fourth, right position family size. In all four contrasts, we matched, as best as we could, on all other properties. We were not able to match for identical constituents as we did in the actual experiments, but we were able to match for the properties of the constituents. Table 1 summarizes the characteristics of the four post hoc contrasts as well as the observed mean reaction times (item means were calculated by subjects). Only the contrast of the left position family frequency yielded a significant difference in reaction times [Contrast $1: t 2(26)=$ $-2.88, p=.008$; Contrast 2: $t 2(28)=-1.94, p=.062]$. The results of both post hoc designs contrasting position family size yielded no significant difference (Contrasts 3 and 4: $t 2<1$ ).

In Contrasts 5 and 6 of Table 1, we compare the lemma frequency of the left and right constituents themselves. Similar to the results of Van Jaarsveld and Rattink (1988), the reaction times for both these contrasts were not significantly different (Contrasts 5 and 6: $t 2<1$ ). In a separate Experiment, ${ }^{7}$ we presented all left and right constituents of the compounds of Experiment $1 \mathrm{a}$ and $1 \mathrm{~b}$ in isolation, as simplex nouns. In this Experiment, both frequency contrasts of Table 1 yielded a significant difference in reaction times [Contrast 5: 526 and $585 \mathrm{~ms}$ respectively: $t 2(34)=$ $-3.13, p=.002$; Contrast 6: 531 and 560 ms respectively: $t 2(36)=-1.74, p=$ .045]. This implies, surprisingly, that the position family frequency of the left constituent plays a facilitatory role while at the same time the frequency of occurrence of this constituent itself when embedded in a compound appears to be irrelevant. We return to this unexpected finding under General Discussion.

Does the morphological family affect the processing of English compounds in the same way? Note that English compounds can be written with either a space between the constituents or with a hyphen or they can be concatenated. The question addressed in Experiment 2 is whether concatenated and open compounds are processed differently as revealed by differing contributions of position family size and position family frequency, as the constituents of open compounds are represented in their orthography as being simplex words.

\section{EXPERIMENT 2}

\section{Method}

Participants. Twenty participants, mostly undergraduates at the State University of New York, Albany, were paid to take part in this experiment or received partial course credits. All were native speakers of English.

Materials. We selected 120 transparent English compounds from the CELEX lexical database. Sixty of these compounds were written with a space between the two constituents and 60 were concatenated. The concatenated and open compounds always shared one constituent, creating 40 pairs such as cornflake and corn bread with identical left constituents and 20 pairs such as tinplate and silver plate with identical

\footnotetext{
${ }^{7}$ We conducted a standard VLD experiment (see the procedure for Experiment 1) except that the size of the letters on the screen for these monomorphemic words was 36 instead of 21 point. Sixteen participants responded to a list consisting of the constituents of Experiment 1a and the pseudoconstituents. Sixteen other participants responded to a list consisting of the constituents of Experiment $1 \mathrm{~b}$ and the pseudoconstituents.
} 
DE JONG ET AL.

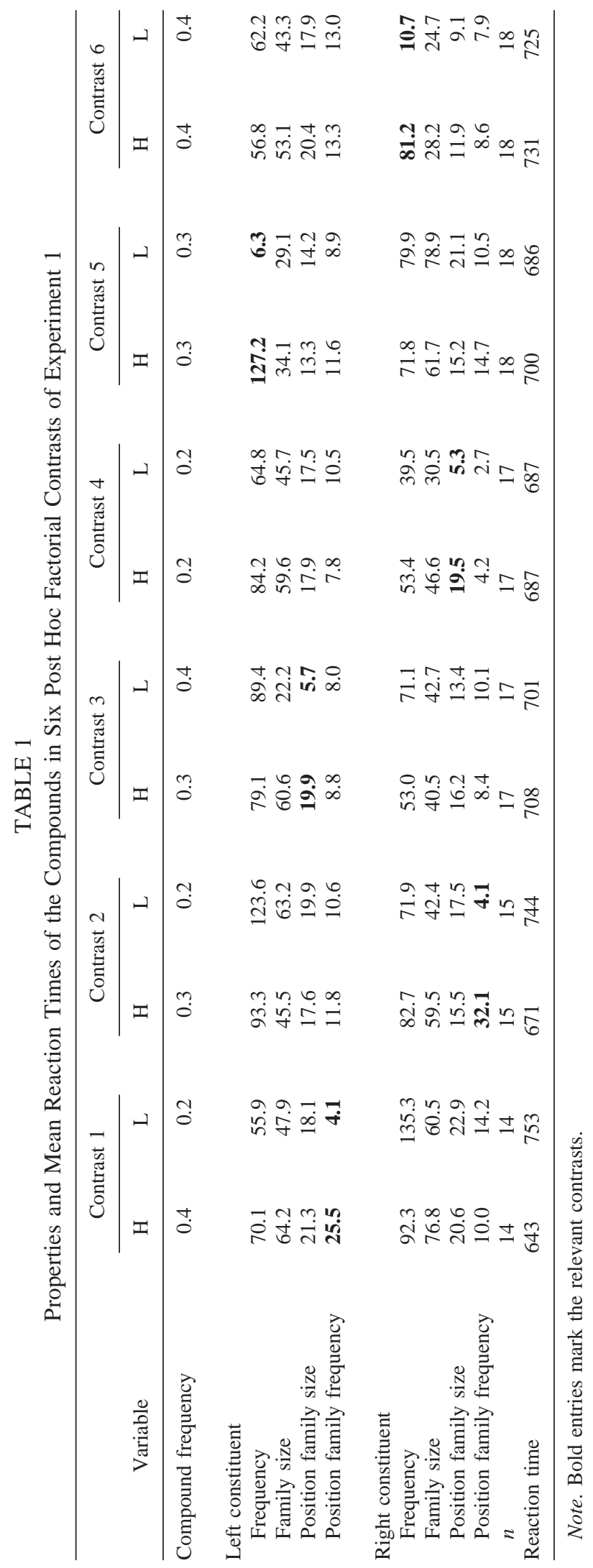


right constituents. The concatenated and open compounds were matched on mean compound frequency [concatenated: $0.66(1.19)$; open: $0.63(1.48)^{8}$ ], mean constituent frequency of the not-shared constituent [concatenated: 88.2 (119.2); open: 103.8 (119.7)], mean family size of the not-shared constituent [concatenated: 14.7 (14.3); open: 12.0 (17.3)], and mean length in letters of the not-shared constituent (concatenated: 4.7; open: 5.1). The properties of the shared constituents were as follows: mean constituent frequency 232.5 (274.7), mean family size 29.7 (31.8), and mean length in letters 4.6.

We constructed two experimental lists such that the same constituents occurred in one list only and such that each list had the same number of concatenated and open compounds. Each compound was paired with a pseudocompound, which consisted of an existing constituent either in the left or right position combined with a pseudoconstituent which did not violate the phonotactical rules of English. In each list, half of the pseudocompounds were open and half of them were concatenated.

Procedure. Participants were tested in noiseproof experimental rooms. They were asked to decide as quickly and accurately as possible whether a letter string appearing on the computer screen was a real English word. Each stimulus was preceded by a fixation mark in the middle of the screen for 500 $\mathrm{ms}$. After $500 \mathrm{~ms}$, the stimulus appeared at the same position. Stimuli were presented on a Power Macintosh 6100/60AV personal computer in black lowercase 21-point Helvetica letters on a white background and they remained on the screen for $1500 \mathrm{~ms}$. The maximum time span allowed for a response was $2000 \mathrm{~ms}$ from stimulus onset. Responses were registered on a Psyscope response box.

\section{Results and Discussion}

One participant performed the experiment with an overall error rate greater than $25 \%$. All data of this participant were excluded from further analyses. The mean reaction time for two compounds (one concatenated and one open compound) exceeded 3 standard deviations from the mean reaction time and were also excluded from further analyses. The open compounds were responded to faster than the concatenated compounds [mean reaction times 747 (119) and 785 (131) ms respectively: $t 1(34)=4.02, p=.000 ; t 2(116)=2.35, p=.020$, using two-tailed $t$ tests] and elicited fewer erroneous responses, although not reliably so [mean error proportions $0.023(0.040)$ and $0.042(0.078)$ respectively; $t 1(34)=1.55, p=.131 ; t 2(116)=$ $1.95, p=.054]$.

In the correlation analysis, the correlation for the concatenated and open compounds between reaction times and compound frequency was identical [for both the concatenated and open compounds $r=-.332 ; t(57)=-2.66, p=.005]$. Comparable to the results of the Dutch compounds, the correlation of the reaction times of the concatenated compounds and left position family frequency was slightly higher $[r=-.328 ; t(57)=-2.62, p=.006]$ than the correlation with left position family size $[r=-.294, t(57)=-2.32, p=.012]$. In a stepwise linear regression analysis, only the compound frequency remains as a reliable predictor of response latencies. The high intercorrelation of left position family size and left position family frequency $[r=.863, t(57)=12.92, p=.000]$ leads to substantial collinearity in the data. We therefore also carried out a nonparametric tree-based regression analysis (Breiman, Friedman, Olshen, \& Stone, 1984), which singles out left position family frequency and compound frequency as reliable predictors. ${ }^{9}$ This suggests that the

\footnotetext{
${ }^{8}$ The CELEX lexical database (Baayen et al., 1995) lists all compounds, but does not provide the frequency of occurrence of the open compounds. In order to obtain comparable frequency counts for the open compounds, we made a list of word bigrams of the corpus used for CELEX and their frequencies. Thus, we obtained the frequency with which a given word, for instance, apple, was followed by another word (e.g., pie). To check whether the concatenated compounds we used were indeed written without spaces in the CELEX corpus, we also calculated the frequency of these concatenated compounds when written with a space. This mean frequency of occurrence was very low: 0.06. The open compounds of this study were never written as concatenated compounds in the CELEX database.

${ }^{9}$ To gauge the independent influence of the two variables left position family size and left position family frequency for the Dutch compounds in Experiment 1, we were able to make post hoc factorial contrasts. As the number of items for the English compounds was substantially reduced, such post hoc factorial contrasts were impossible to make.
} 
English concatenated compounds are processed in a similar way as the Dutch compounds. Turning to the English open compounds, however, we see a different pattern. For the English open compounds, we find that the correlation between reaction times and left position family frequency is lower $[r=-.290, t(57)=-2.29, p=.013]$ than the correlation for left position family size $[r=-.350, t(57)=-2.82, p=$ .003]. A stepwise linear regression analysis as well as a nonparametric tree-based regression analysis single out left position family size and compound frequency as reliable predictors. ${ }^{10}$ For both the concatenated and open compounds, there were no reliable effects for the right constituents.

Although English concatenated and open compounds are processed similarly with respect to their full form (both kinds show an equal effect of compound frequency), the present results suggest that the left constituents of English open compounds are processed differently than the left constituents of concatenated compounds. Whereas the processing of constituents of concatenated compounds (of English as well as of Dutch compounds) is influenced by position family frequency, the processing of left constituents of open compounds is influenced by position family size. This type effect of the morphological family is comparable to the family size effect of simplex words, suggesting that left constituents of English open compounds are processed more similarly to simplex words than is the case for constituents of concatenated compounds. We return to this issue under General Discussion.

In Experiment 3, we investigate whether the difference between concatenated and open compounds in English can be interpreted as a superficial effect of the orthography by inserting an artificial space between constituents of Dutch compounds. If the different effect found for English concatenated and open compounds is due to a superficial orthographic effect, we should be able to induce a similar difference for Dutch concatenated and (artificial) open compounds.

\section{EXPERIMENT 3}

\section{Method}

Participants. Twenty-four participants, mostly undergraduates at Nijmegen University, were paid to take part in this experiment. All were native speakers of Dutch.

Materials. We used the same compounds and pseudocompounds as in Experiment 1a. We added a space between the constituents of the compounds and pseudocompounds.

Procedure. The procedure was identical to that of Experiment 1a, except that we asked the participants to decide as quickly as possible whether the two letter strings appearing on the screen were real Dutch words.

\section{Results and Discussion}

All participants performed the experiment with an overall error rate less than $11 \%$. The reaction times in this experiment are longer than the reaction times of Experiment $1 \mathrm{a}$, in which these compounds were presented without the artificial space [739 and 679 ms respectively; $t 1(94)=3.35, p=.000 ; t 2(218)=4.66, p=.000$, using twotailed $t$ tests].

A correlation analysis showed that, although the inserted space changed the standard visual form of the compound, the correlation between full-form frequency (compound frequency) and reaction times was still highly significant $[r=-.376$,

${ }^{10}$ Some English compounds bear stress on the second constituent rather than on the first constituent (apple ṕie versus śide walk). In our data, 11 open compounds had primary stress on the second constituent. Excluding these open compounds from the correlational analyses did not alter the pattern of results. 
$t(108)=-4.21, p=.000]$. Comparable to the results of Experiment 1a, in which these compounds were written without an artificial space, the correlation between reaction times and left position family frequency $[r=-.257, t(108)=-2.76$, $p=.003]$ was higher than the correlation with left position family size $[r=-.180$, $t(108)=-1.91, p=.030]$. A partial correlation of the left position family frequency, after partialing out the influence of left position family size, showed a significant correlation $[r=-.198, t(107)=-2.09, p=.019]$, but the partial correlation of left position family size, after partialing out the influence of left position family frequency, was not significant $(r=.071, t<1)$. Similarly, a tree-based analysis ranked left position family frequency above left position family size. These results suggest that although the space between the constituents made the lexical decision task more difficult, as evidenced by the longer reaction times in this experiment in comparison to the reaction times of Experiment 1a, the artificial space had no clear effect on the way in which these compounds were processed. Similar to Dutch concatenated compounds and contrary to English open compounds, the processing of Dutch artificial open compounds is influenced by a token effect of the morphological family. We generalize from these results in Dutch that the different contributions of family size and family frequency for concatenated and open compounds in English cannot be attributed to the mere presence of a space between the constituents of a compound in the visual input.

If the difference in processing of English concatenated and open compounds is not due to some superficial effect of the orthography, but rather due to a difference at a deeper level of processing, this suggests that open compounds might be, at a central level, represented in a different way than concatenated compounds. In the three subexperiments of Experiment 4, we investigate this possibility by presenting English simplex words, which are factorially contrasted with respect to the number of different kinds of family members (concatenated, hyphenated, and open family members) while keeping the total family size constant.

\section{EXPERIMENT 4}

\section{Method}

Participants. Twenty participants, mostly undergraduates at the State University of New York, Albany, were paid to take part in these experiments or received partial course credits. All were native speakers of English.

Materials of experiment 4a. We selected 34 simplex English nouns divided into two groups of 17 words each, using the CELEX lexical database. These two groups of words were matched on total family size [means: $17.6(8.4)$ and $15.9(7.6)]$ but differed in the proportion within this family of concatenated family members versus the sum of open and hyphenated family members. Seventeen words had a high number of concatenated family members [mean: 15.4 (6.3)] and 17 contained only a few concatenated family members [mean: $2.6(1.8)$ ]. Note that by contrasting the concatenated family members and at the same time keeping the total family size constant, we also contrasted the sum of open and hyphenated family members together (in the high condition 2.2 and in the low condition 13.3.) In addition to the total family size, the words were also matched on frequency [high: 104.3 (125.6); low: 113.5 (131.1)] and mean length in letters (high: 5.5; low: 4.8).

Materials of experiment $4 b$. We selected 34 simplex English nouns, using the CELEX lexical database, divided into two groups of 17 words each. These two groups of words were matched on total family size [means: 36.1 (23.8) and 35.4 (22.7)] but differed in the proportion within this family of family members written with a hyphen versus concatenated or open family members. Seventeen words had a high number of hyphenated family members [mean:19.4 (21.2)] and 17 contained only a few hyphenated family members [mean:1.2(1.8)]. Note that by contrasting the number of hyphenated family members while keeping the total family size constant, we at the same time contrasted the sum of concatenated and open family members (in the high condition 16.7 and in the low condition 34.2). In addition 
to the total family size, the words were matched on frequency [high: 133.2 (120.7); low: 134.9 (126.2)] and mean length in letters (high: 4.2; low: 4.4).

Materials of experiment $4 c$. We selected 48 simplex English nouns from the CELEX lexical database. We divided these into two groups of 24 words each matched on total family size [means: 30.7 (21.5) and 31.0 (22.0)]. The first group had a high number of open family members within this family [mean: 11.5 (5.1)] and within the families of the second group only a few family members were written with a space [mean: $1.3(1.3)$ ]. Note that by contrasting the number of open family members within the family while keeping the total family size constant, we also contrasted the number of concatenated and hyphenated family members jointly (high condition: 19.2; low condition: 29.7). In addition to matching for the total family size, we also matched the two groups of words on frequency [high: 109.0 (108.5); low: 106.4 (98.2)] and mean length in letters (high: 4.6; low: 4.2).

We combined all simplex nouns of Experiments $4 \mathrm{a}$ to $4 \mathrm{c}$ into one list, in which the targets of one experiment served as the fillers of another experiment. Each word was paired with a pseudoword which did not violate the phonotactical rules of English.

Procedure. The procedure was identical to that of Experiment 2, except that the size of the letters appearing on the screen for these monomorphemic words was 36 instead of 21 point.

\section{Results and Discussion}

All participants performed the experiment with an overall error rate less than $11 \%$. One word in Experiment $4 \mathrm{a}$, centre, was excluded from further analysis because its spelling did not conform to the American spelling conventions (resulting in a high error score and long reaction times). The reaction times of three words (one from Experiment $4 \mathrm{a}$ and two from Experiment $4 \mathrm{~b}$ ) exceeded 3 standard deviations from the mean reaction time and were also excluded from further analysis. Table 2 shows the mean reaction times (calculated over the correct responses) and error scores (calculated over all responses) for the two experimental conditions of all three subexperiments. We used two-tailed $t$ tests as we have no a priori hypothesis concerning the direction of potential differences. The difference in reaction times and error scores of Experiment 4a [reaction times: $t 1, t 2<1$; error scores: $t 1(19)=-1.64, p=$ $.117, t 2(30)=-1.09, p=.286]$ and Experiment $4 \mathrm{~b}$ (reaction times: $t 1 \approx 1, t 2<$ 1 ; error scores: $t 1, t 2<1$ ) were not significant. The difference in reaction times of Experiment 4c, however, was significant. The words with a low number of open family members were responded to faster than those with a high number of open family members $[t 1(19)=2.93, p=.009 ; t 2(46)=2.89, p=.006]$. There were no reliable differences in the error scores $(t 1, t 2<1)$. Using Bonferroni adjustments, we find that, across the three subexperiments, the result of Experiment $4 \mathrm{c}$ remains significant at the $5 \%$ level.

Combining these three experiments, we see the same pattern in a post hoc correla-

TABLE 2

Results of Experiment 4

\begin{tabular}{|c|c|c|c|c|}
\hline & RT & $(S D)$ & Error & $(S D)$ \\
\hline \multicolumn{5}{|c|}{$\langle$ Experiment $4 \mathrm{a}\rangle$} \\
\hline High amount concatenated family members & 577 & (104) & 0.00 & $(0.01)$ \\
\hline Low amount concatenated family members & 586 & (98) & 0.01 & $(0.03)$ \\
\hline \multicolumn{5}{|c|}{$\langle$ Experiment $4 \mathrm{~b}\rangle$} \\
\hline High amount hyphenated family members & 556 & $(101)$ & 0.01 & $(0.03)$ \\
\hline Low amount hyphenated family members & & $(84)$ & 0.02 & $(0.04)$ \\
\hline High amount open family members & 586 & (101) & 0.00 & $(0.01)$ \\
\hline Low amount open family members & 561 & (92) & 0.01 & $(0.02)$ \\
\hline
\end{tabular}

Note. Means and standard deviations of response latencies and error proportions (by participants) are shown. 
tion analysis. If we count the family size of only the concatenated family members, we obtain a marginally significant negative correlation with reaction times $[r=$ $-.200, t(93)=-1.96, p=.053$ ]. Counting only the hyphenated family members results in a similar correlation with reaction times $[r=-.185, t(93)=-1.82, p=$ .073]. But restricting the count to only the open family members yields no reliable correlation $(r=-.004, t<1)$. The best correlation was obtained if the family count was based on the sum of concatenated and hyphenated family members together $[r=-.306, t(93)=-3.10, p=.003]$. Just as shown by the reaction times of the factorial designs, these correlation analyses indicate that open family members do not contribute to the effect of family size of a monomorphemic word.

We inspected the family members of the words in these experiments with respect to frequency. The concatenated family members had the highest mean frequency (9.7), but the mean frequencies of the hyphenated (0.3) and open (0.4) family members were not reliably different $(t<1)$. This eliminates the possibility that merely the (low) frequency of these family members would account for the difference in the effects of family size: The open family members were just as (in-)frequent as the hyphenated family members, but only the latter showed a reliable correlation with reaction times.

It is well known that there is variability in the orthography of compounds with respect to spaces, hyphens, or concatenation. Diachronically, phrases which started out with spaces between the words, but which became very common, can now be written as one single word. Synchronically, variation in spelling of compounds is also apparent. In our materials, we checked the variability in spellings of the family members. Of all 2254 family members of the items in Experiment 4a to 4c, a total of 433 family members also occurred with an alternative spelling. Most of these were hyphenated family members for which the alternative spelling was with a space rather than a hyphen. Of these 433 family members, the frequency of occurrence of the alternative spelling was higher than the frequency of the regular spelling for 174 of these family members. Changing the status (concatenated, hyphenated, or open) of the family members according to these frequencies, such that the highest frequency of the different spellings decides the status, did not change the pattern of results.

\section{GENERAL DISCUSSION}

This study addresses the processing and representation of compounds in Dutch and English using various measures relating to the morphological family as diagnostics. For simplex words and for stems in derived words the family size, a type count of the morphological family codetermines response latencies. Experiment 1 revealed that for constituents in Dutch compounds, such a type count does not predict response latencies. Instead, the position family frequency predicts decision latencies.

Constituents of Dutch compounds are always concatenated, but in English, compounds can be written in three different ways: concatenated, hyphenated, or with a space between the constituents. In Experiment 2, we compared English concatenated and open compounds. Similar to the Dutch results of Experiment 1, English compounds (concatenated and open) show an effect of compound frequency. For the English concatenated compounds, again similar to the Dutch results of Experiment 1 , the position family frequency was a better predictor than the position family size. For the English open compounds, by contrast, the effect of position family size was more important. This outcome suggests that the presence of a space between the constituents of open compounds renders the processing of the constituents more comparable to the processing of simplex words, as simplex words also show a type effect of the morphological family rather than a token effect. 
In Experiment 3, we inserted an artificial space between Dutch compounds (see also Inhoff, Radach, \& Heller, 1999). Comparable to the results of the Dutch concatenated compounds, and contrary to the results of the English open compounds, these Dutch artificial open compounds showed, in addition to an effect of compound frequency, an effect of position family frequency only. This suggests that the differential effects of the English open and concatenated compounds are not due to a superficial effect of the orthography. Converging evidence for the central level of the observed difference between concatenated and open compounds comes from Experiment 4. In this Experiment, English simplex words were presented. The results of Experiment $4 \mathrm{c}$ and the post hoc correlations of all the combined Experiments $4 \mathrm{a}$ to $4 \mathrm{c}$ showed that for English simplex words, only the number of concatenated and hyphenated family members plays a role. The more concatenated and hyphenated family members a word has, the faster participants are able to respond. The number of open family members, however, appears to be irrelevant.

Our hypothesis is that the observed effect of position family frequency of concatenated compounds in Dutch and English taps into a selection process based on conditional constituent probabilities. To see this, consider as an example the mini-lexicon of Table 3. Imagine that this lexicon represents all compounds. The effect of position family frequency of any compound with the left constituent molen can now be formulated as follows, keeping in mind that our participants knew that a target word in Experiments 1-3 would always be a compound. Conditional on the set of compounds, the probability that a compound has molen as its left constituent is 40/100. Apparently, participants made use of this kind of position-dependent probabilities to speed up their responses.

Recall that the constituent frequencies of both the left and right constituent themselves are irrelevant, but that the frequency of the compound as a whole does play a role. This seems to suggest that compounds are processed as wholes and that their morphological structure does not play a role. However, although the "unigram", frequency of the constituents is irrelevant, the existence of the effect of the positional family frequency, a conditional "bigram" probability, shows that lexical processing is nevertheless sensitive to morphological structure. We interpret this conditional "bigram" effect to occur peripheral to the central lexicon, at the level of the access representations, possibly even affecting eye movements (Hyönä \& Pollatsek, 1998).

The differential effect for the English open compounds, as well as the finding that open compounds do not contribute to a simplex word's family, leads to an interesting question. Surprisingly, the orthographical space between the constituents in English leads to different processes for recognizing the compound itself, and to a differential status in the central mental lexicon of these kinds of words. Possibly, the open compounds are phraselike entities and therefore fall outside the morphological family of a simplex word. The question which arises in this context is whether the phraselike

TABLE 3

Artificial Mini-Lexicon

\begin{tabular}{lc}
\hline Lemma & Frequency \\
\hline molenwiek & 10 \\
molensteen & 20 \\
molenrad & 10 \\
windmolen & 20 \\
windvaan & 10 \\
driehoek & 30 \\
Total & 100 \\
\hline
\end{tabular}


status of these compounds has led to writing a space between the constituents or whether the space in the orthography has led to the phraselike status. Whatever the direction of causality may turn out to be, the fact remains that, apparently, orthographic phrases are processed and stored differently from orthographic words.

\section{REFERENCES}

Baayen, R. H., Dijkstra, T., \& Schreuder, R. (1997). Singulars and plurals in Dutch: Evidence for a parallel dual route model. Journal of Memory and Language, 36, 94-117.

Baayen, R. H., Lieber, R., \& Schreuder, R. (1997). The morphological complexity of simplex nouns. Linguistics, 35, 861-877.

Baayen, R. H., Piepenbrock, R., \& Gulikers, L. (1995). The CELEX lexical database (CD-ROM). Philadelphia: Linguistic Data Consortium, University of Pennsylvania.

Bertram, R., Baayen, R. H., \& Schreuder, R. (2000). Effects of family size for complex words. Journal of Memory and Language, 42, 390-405.

Bertram, R., Schreuder, R., \& Baayen, R. H. (2000). The balance of storage and computation in morphological processing: The role of word formation type, affixal homonymy, and productivity. Journal of Experimental Psychology: Learning, Memory, and Cognition, 26, 419-511.

Breiman, L., Friedman, J. H., Olshen, R., \& Stone, C. J. (1984). Classification and regression trees. Belmont, CA: Wadsworth International Group.

De Jong, N. H., Schreuder, R., \& Baayen, R. H. (2000). The morphological family size effect and morphology. Language and Cognitive Processes, 15, 329-365.

Hyönä, J., \& Pollatsek, A. (1998). Reading finnish compound words: Eye fixations are affected by component morphemes. Journal of Experimental Psychology: Human Perception and Performance, 24, $1612-1627$.

Inhoff, A. W., Radach, R., \& Heller, D. (2000). Complex compounds in german: Interword spaces facilitate segmentation but hinder assignment of meaning. Journal of Memory and Language, 42, $23-50$.

Marslen-Wilson, W. (in press). Access to lexical representations: Cross-linguistic issues. Language and Cognitive Processes.

Sandra, D. (1990). On the representation and processing of compound words: Automatic access to constituent morphemes does not occur. Quarterly Journal of Experimental Psychology, 42A, 529-567.

Schreuder, R., \& Baayen, R. H. (1997). How complex simplex words can be. Journal of Memory and Language, 37, 118-139.

Taft, M. (1979). Recognition of affixed words and the word frequency effect. Memory \& Cognition, 7, 263-272.

Taft, M., \& Forster, K. I. (1976). Lexical storage and retrieval of polymorphemic and polysyllabic words. Journal of Verbal Learning and Verbal Behavior, 15, 607-620.

Van Jaarsveld, H., \& Rattink, G. (1988). Frequency effects in the processing of lexicalized and novel nominal compounds. Journal of Psycholinguistic Research, 17, 447-473.

Zwitserlood, P. (1994). The role of semantic transparency in the processing and representation of Dutch compounds. Language and Cognitive Processes, 9, 341-368. 